\title{
Lire des documents composites en histoire scolaire : problème de lecture et problème historique
}

Reading composite documents in school history: reading problem and historical problem

\section{Sylvain Doussot}

\section{OpenEdition \\ Journals}

\section{Édition électronique}

URL : http://journals.openedition.org/pratiques/8411

DOI : $10.4000 /$ pratiques.8411

ISSN : 2425-2042

\section{Éditeur}

Centre de recherche sur les médiations (CREM)

\section{Référence électronique}

Sylvain Doussot, «Lire des documents composites en histoire scolaire : problème de lecture et problème historique », Pratiques [En ligne], 185-186 | 2020, mis en ligne le 30 juin 2020, consulté le 15 octobre 2020. URL : http://journals.openedition.org/pratiques/8411 ; DOI : https://doi.org/10.4000/ pratiques. 8411

Ce document a été généré automatiquement le 15 octobre 2020.

(c) Tous droits réservés 


\title{
Lire des documents composites en histoire scolaire : problème de lecture et problème historique
}

Reading composite documents in school history: reading problem and historical problem

\author{
Sylvain Doussot
}

1 Le travail documentaire fait partie de l'essence même de l'approche historique du passé qui constitue une connaissance "par traces » (Bloch, 1997 [1949]), ces dernières étant par nature composites. Il est largement transposé en histoire scolaire à travers l'étude de documents, et notamment dans la visée d'analyse des différentes composantes des documents, susceptible de fournir les moyens d'une double critique par confrontation de ce que dit le document et de ce qu'il est. Cependant, l'observation des pratiques disciplinaires scolaires dominantes montre, dans nombre de recherches didactiques, que cette transposition modifie radicalement la nature du travail de lecture des documents composites par les élèves par rapport à la visée scientifique, et ouvre à l'identification des enjeux épistémologiques des difficultés qu'ils rencontrent. En s'appuyant sur l'historiographie et l'épistémologie de l'histoire il est possible de mettre au jour des conditions disciplinaires de gestion de l'hétérogénéité des registres énonciatifs et des systèmes sémiotiques lors de l'acte de lecture de ces documents. Sous l'angle d'une didactique de l'histoire ainsi armée épistémologiquement, et en appui sur quelques études de cas, la présente étude identifie trois échelles d'appréhension de cette hétérogénéité : au sein d'une même trace, par confrontation de traces diverses, et selon les relations que ces traces entretiennent avec une même situation historique. Cette troisième échelle, proprement disciplinaire, indexe les enjeux sémiotiques et énonciatifs des deux premières à la construction d'un problème historique scolaire. Autrement dit, il serait plus aisé pour les élèves de lire des documents composites lorsque ces derniers constituent des traces d'une même situation socio-historique. 


\section{Un usage courant des documents composites en classe d'histoire}

2 L'histoire scolaire a suivi l'évolution de sa référence scientifique - sur ce plan tout au moins - et prend en charge des documents de nature extrêmement variée, qui vont de l'archive écrite dans sa forme la plus traditionnelle (une archive d'armateur d'un navire négrier du XVII e siècle par exemple) à des monuments (au cours de visites ou par le biais de photographies), des témoignages oraux et des objets (mis au jour par l'archéologie). Elle peut même utiliser des documents spécifiquement scolaires que l'historien ne peut considérer : des représentations de situations du passé sous forme de film de fiction, de docu-fiction ou encore de bandes dessinées (liste non exhaustive). Une telle diversité conduit à la confrontation des élèves à des formes multiples de documents dont une des caractéristiques est qu'ils sont composés de différents éléments parfois hétérogènes. Une image telle qu'une allégorie prérévolutionnaire (figure 1) contient du texte inséré (sur la pierre il est écrit : « Taille, Impôts et Corvées ») et lorsqu'elle est dans un manuel elle est accompagnée d'une légende (par exemple : « gravure de 1787 »).

Figure $1:$ un exemple de document image

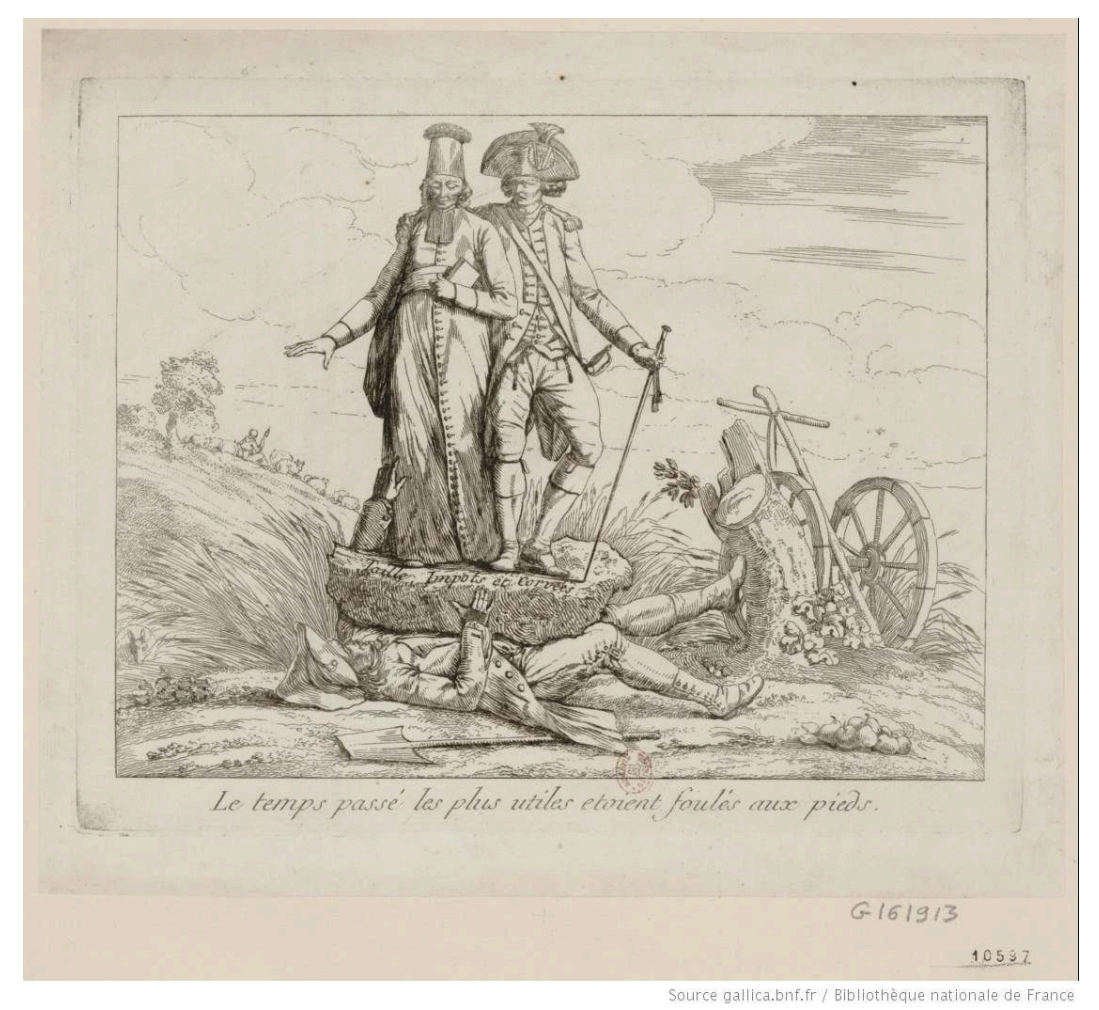

Au sein du manuel elle est positionnée dans la page de droite parmi un ensemble de documents divers (textes, reconstitutions dessinées, photographies d'objets ou de monuments, etc.) légendés, accompagnés parfois de questions, et en vis-à-vis avec une page de gauche qui contient généralement un texte de « cours » qui synthétise le savoir historique sur l'évènement ou la période considérée. L'usage dominant du manuel en classe est cependant ponctuel : l'enseignant renvoie les élèves à un document ou quelques documents, mais rarement à un ensemble structuré dans le manuel. Le manuel, comme support, est concurrencé par la production par les enseignants de 
fiches d'études de documents composés par eux-mêmes, mais sur le même modèle (en général en noir et blanc), et par l'usage du vidéoprojecteur (qui permet la couleur).

4 La dimension composite de documents soumis à la lecture des élèves de classe d'histoire se révèle une source de difficultés pour eux dans les études qui s'intéressent aux enjeux didactiques de ces activités d'étude de documents. Ainsi en est-il de l'étude de la figure 1 en classe de CM2 (Le Marec, 2010) dans une situation inhabituelle pour les élèves : un temps long est consacré à un travail de description de l'image sur la seule consigne "décris l'image ", d'abord individuellement, puis par une mise en commun très progressivement guidée par l'enseignante. L'analyse des productions orales des élèves indique que l'allégorie n'est pas comprise par les élèves qui appliquent à l'image une lecture réaliste qui se focalise sur les détails (bergère et moutons en arrière-plan) et voient essentiellement une scène de bataille (la charrette est ainsi prise pour un canon, la position des trois personnages pour une violence de combat guerrier). Plus généralement, l'image est décomposée par les élèves qui y prélèvent des informations disparates lorsqu'elles prennent un sens possible pour eux (on le voit nettement dans le prélèvement des mots « impôts » et « corvées » et le fait qu'ils ignorent tous « taille »). Ils n'appréhendent pas le système sémiotique de l'allégorie et restent dans un système réaliste qui correspond à l'habitude disciplinaire de ces élèves de fin de primaire, qui les conduit au prélèvement d'informations (Tutiaux-Guillon, 2008) sans souci de la forme sémiotique portée par le document en tant qu'entité. Parallèlement, les registres énonciatifs en jeu - la légende ("gravure de 1787 »), l'image et le texte inscrit sur la pierre de l'image - ne sont pas davantage pris en charge par la lecture des élèves.

\section{Une interprétation épistémologique des difficultés de lecture des élèves}

5 Les approches didactiques fondées sur des enjeux épistémologiques de la discipline histoire interprètent cette indifférence aux différences sémiotiques et énonciatives dans l'activité de lecture des documents composites par l'absence de prise en compte de la double situation en jeu dans l'étude de sources du passé: situation d'étude actuelle et situation passée étudiée. Dans l'activité experte des historiens, cette dualité est médiée par la trace du passé accessible dans le présent. Pour interpréter les constats empiriques il est nécessaire de distinguer la trace au sens propre du terme ; la source qui est une trace utilisée dans une enquête singulière, guidée par des questions et des hypothèses et dans laquelle on recherche des indices; et enfin le document qui porte de manière plus ou moins directe ${ }^{1}$ la trace à la connaissance du lecteur. Par ces distinctions épistémologiques on est amené à bien différencier deux niveaux de lecture si l'on souhaite identifier les enjeux de leur articulation du point de vue disciplinaire. D'une part la lecture du document pour elle-même, qui suppose la mise ne relation de ce qu'est le document et de ce qu'il dit, et d'autre part la lecture du document pour produire et vérifier l'explication d'un évènement passé. La pratique historienne ainsi analysée théoriquement consiste à articuler ensemble ces différentes dimensions de l'activité de lecture documentaire : «ma dissociation entre l'établissement des faits par la méthode critique et leur interprétation ultérieure, si elle répond aux contraintes effectives de l'enseignement et de la synthèse, n'est pas soutenable logiquement " (Prost, 1996, p. 73). 
Dans les pratiques effectives de l'enseignement de l'histoire, le premier niveau de lecture est pris en charge par le travail de distinction entre légende et contenu, c'est-àdire entre la critique externe du document (ce qu'il est et ce qu'il fait dans le passé, dans son contexte) et sa critique interne (ce qu'il dit du passé, du contexte). Cependant, cette distinction exercée de manière quasi-systématique jusqu'en fin de lycée par le biais de questions rituelles sur la légende puis sur le contenu, ne permet pas réellement de construire la relation entre les deux registres énonciatifs externes et internes. S'agissant du second niveau de lecture, sa mise en relation avec le niveau proprement documentaire est encore plus délicate. Bien souvent l'étude des documents vise à fournir des informations illustrant partiellement le récit explicatif final de l'évènement ou du phénomène passé en jeu, parce que ce dernier est d'une ampleur telle qu'il ne peut être produit par la seule étude de quelques documents, et parce que le questionnement est émietté et ne renvoie pas à un problème de connaissance du passé identifiable (Fabre, 2007, p. 75). Ce faisant, les activités de lecture de documents ne permettent pas aux élèves de côtoyer la différenciation entre information et indice, comme le montre une étude comparative en primaire et secondaire (Cariou, 2016).

7 Tout espoir n'est cependant pas perdu puisque cette même étude de D. Cariou montre que par moment, au sein même de ces activités dominantes, les élèves sont en mesure d'entrer dans une approche indiciaire qui leur permet de prendre en charge une partie des enjeux de lecture historique des documents composites. Dans cette double étude de cas qui porte elle aussi sur une allégorie publiée avant la Révolution française, D. Cariou pointe ainsi des échanges qui renvoient à un jeu d'apprentissage indiciaire. Ce dernier se révèle notamment lorsque l'enseignante régule l'activité de lecture de l'image par des questions qui ciblent des détails de la scène qu'elle sait significatifs des savoirs en jeu pour identifier un sens politique à l'image (en particulier la distinction des trois ordres dans les trois personnages pour expliquer leur position). Selon les objets sur lesquels portent la réticence et l'expression de l'enseignante, une recherche peut s'opérer en direction d'une lecture qui dépasse le stade de la simple accumulation d'informations.

8 Ces capacités manifestées par certains élèves en situation habituelle d'étude de document, en fonction des interventions de l'enseignant, sont également mises en lumières par des travaux non disciplinaires dans le champ de l'informationcommunication. C'est notamment le cas en ce qui concerne le premier niveau de lecture (mise en relation entre dimension externe et interne du document) comme le montre la notion de "confiance épistémique» envers un document analysé par A. Tricot, G. Sahut, \& J. Lemarié (2016, p.99). Celle-ci « est un enjeu lorsque nous sommes amenés à croire les autres pour acquérir des informations sur le monde ». Dans les cas d'histoire scolaire considérés ici, cette confiance se porte sur l'auteur du document comme élément externe, pour relativiser la qualité de l'information que fournit ce dernier (dimension interne). Mais chez les jeunes étudiés par N. Boubée \& A. Tricot (2011, p. 267), « les critères utiles à l'évaluation de la "qualité" (validité du contenu et autorité de la source) sont très faiblement présents sauf lorsque les jeunes disposent de connaissances solides sur le thème de recherche». Les deux auteurs concluent alors non pas à l'absence d'« esprit critique » au sens général du terme, mais à la dépendance de cette capacité critique envers la maitrise de connaissances. Cette nécessité identifiée de connaissances du domaine dans lequel s'opère la recherche documentaire fait écho à la relation entre le premier et le second niveau de lecture des 
documents, entre la critique du document et la critique de l'explication du passé sur lequel le document peut informer. Comment contextualiser le document sans connaissance du contexte? Mais réciproquement comment connaitre le contexte en dehors de l'étude d'éléments singuliers qui le compose ${ }^{2}$ ?

\section{Lecture de documents composites au prisme des spécificités de l'histoire}

9 À première vue, un tel principe conduit à une aporie didactique, comme le formule A. Prost lorsqu'il envisages les enjeux de l'activité de lecture des traces chez les historiens: passons aussi sur l'impossibilité logique de commencer pratiquement l'histoire par la critique des traces. L'exposé classique de la méthode historique qui place la critique au fondement logique de l'édifice exige de telles compétences de l'historien qui prétend critiquer un document que la tâche s'avère impossible à qui n'est pas déjà historien. Il faut y revenir :

La critique procède par comparaison et il est impossible de déceler qu'un document est faux si l'on ignore comment un document vrai aurait dû se présenter [...] Seul un historien aguerri est à même de pratiquer la critique. Ce que confirment les difficultés des étudiants devant les commentaires de texte qui les rassurent, en leur évitant le vertige de la feuille blanche, mais qui s'avèrent, d'expérience commune de correcteur, beaucoup plus difficiles que les dissertations. (Prost, 1996, p. 74)

10 S'il faut déjà être historien pour faire de l'histoire et si des étudiants peinent à entrer dans la pratique documentaire attendue, comment imaginer un enseignement scolaire de la lecture critique de documents composites en histoire? On peut se rassurer tout d'abord par le détail de l'affirmation d'A. Prost, qui confesse au détour d'une phrase que cette conviction est fondée sur une expérience de correcteur et non sur des recherches didactiques. Que disent ces dernières de cette évidence d'expérience?

11 Bien souvent, les thèmes étudiés en histoire scolaire portent sur des phénomènes sur lesquels les élèves ont des connaissances sociales plus ou moins directes. De ce point de vue, la situation prérévolutionnaire et l'allégorie (figure 1) peuvent apparaitre particulièrement difficiles à appréhender, mais ce n'est pas le cas de l'étude de la Première Guerre mondiale. Celle-ci, pourtant jamais étudiée auparavant par des élèves de $\mathrm{CM} 2$, peut s'appuyer sur une représentation déjà non négligeable de ce phénomène anthropologique qu'est la guerre. Ainsi ${ }^{3}$, des élèves de 10/11 ans sont en mesure de modifier leur lecture d'une lettre de soldat à sa famille lorsque l'enseignante questionne au détour d'une échange oral sur le fait que ce soldat disait ou non " tout " dans sa lettre (Doussot \& Vézier, 2014). La question déclenche la prise en compte de la légende de la lettre (qui situe l'auteur comme père de famille) et la mise en relation avec d'autres documents fournis dans le dossier documentaire (notamment le journal de sa section qui décrit militairement les opérations menées, selon un système sémiotique non linéaire; voir figure 2). 
Figure 2 : journal de section du soldat de la Première Guerre

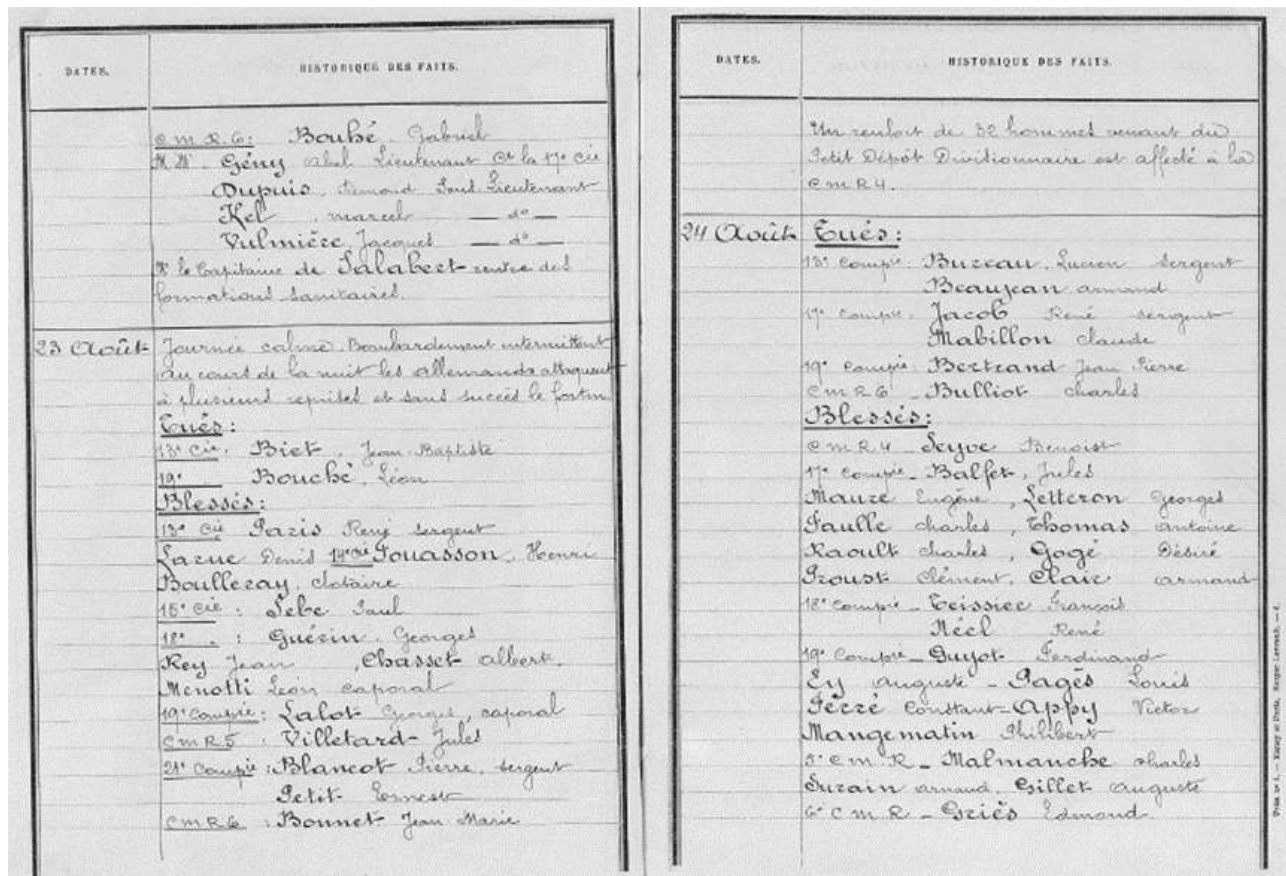

Mais cette appréhension explicite de la nature épistolaire du document initialement laissée de côté par les élèves conduit aussi à la formulation immédiate d'une explication à cette autocensure du soldat : les élèves puisent dans leur connaissance de la guerre pour fournir des hypothèses telles que la « honte » d'évoquer sa situation ou encore la "volonté d'oublier» ce qui est traumatisant. On discerne dans la mise en relation explicite de la légende (nature du document, destinataires, etc.) et du contenu de la lettre, la manière dont s'ouvre alors l'accès aux différentes composantes du dossier documentaire et surtout à leur mise en relation productive dans l'expansion du sens donné à la situation (non seulement le soldat souffre et a peur - ce que les élèves savent déjà de la guerre - mais il souffre de la honte et de ne pouvoir raconter tout ce qu'il vit).

13 Cette étude de cas conduit à mieux envisager le dépassement de l'aporie formulée par A. Prost en prenant en compte les connaissances d'expérience des élèves sur le monde social et les comportements humains parce qu'elles rendent possible la figuration des situations vécues. Si l'on revient au thème du déclenchement de la Révolution française, une étude de document structurée par l'analyse d'un film de fiction ${ }^{4}$ (Doussot, 2017) révèle les connaissances sociales des élèves de CM2 qui leur permettent de mieux saisir les différentes composantes du film: les intentions des auteurs sur le plan externe, et les personnages secondaires de l'intrigue mais essentiels de la situation révolutionnaire comme le colporteur qui diffuse l'information de la prise de la Bastille de village en village. Les élèves sont d'emblée capables d'expliquer la révolte des paysans par leur situation misérable et injuste, qu'ils rapportent analogiquement à des situation vécues ou observées dans leur quotidien selon un modèle explicatif du type " misère et injustice entrainent la révolte ». Les élèves peuvent ensuite requestionner le document dans ses différentes dimensions à condition que ces premières causes de la révolte soient posées comme insuffisantes : par exemple, si la misère et l'injustice sont des causes, comment expliquer qu'elles n'agissent pas avant l'été 1789 alors qu'elles sont déjà présentes? Cette insuffisance du modèle explicatif initial conduit à une 
lecture plus approfondie des registres énonciatifs multiples portés par les personnages du film et d'abord ignorés, parce qu'elle situe leurs actions à une échelle humaine à laquelle les élèves parviennent à se figurer leurs choix et leurs incertitudes. L'étude de la lecture de la figure 1 par des élèves de CM2 (Le Marec, 2010), même si elle pointe les difficultés des élèves à comprendre l'enjeu de contestation de la société d'ordres, montre comment ils mobilisent cependant leurs connaissances du monde sociale pour entrer dans l'interprétation de l'image. L'attention aux détails, que tend à rejeter le professeur, traduit pourtant des intentions de l'auteur de l'image: le calme que manifeste la bergère en arrière-plan contredit l'interprétation d'un conflit guerrier au premier plan selon la lecture réaliste des élèves. «Y'en a qui disent que c'est une guerre mais à mon avis c'est pas une guerre parce là, on voit la femme derrière, elle est toute tranquille avec ses moutons " argumente ainsi une élève : celle-ci se représente une scène de vie à laquelle elle peut donner du sens (Le Marec, 2010, p. 129). De la même manière, le double registre énonciatif du document (image/texte) pourrait être utilisé, dans une régulation enseignante moins ouverte, pour pointer que l'image n'est pas seulement réaliste (rien n'est écrit sur les pierres dans la réalité d'une telle scène de vie rurale).

14 Ce type de potentialité, que nous développons après, et ces constats empiriques de possibilités didactiques de faire entrer les élèves dans une lecture critique des documents composites par appui sur leurs connaissances sociales traduisent un point commun. Ces connaissances nécessaires et disponibles des élèves s'inscrivent dans un questionnement à une échelle historiquement pertinente pour donner du sens aux documents. Dans ces moments de lecture pertinente est en jeu, non seulement un savoir de la réponse (un savoir sur le document), mais également un savoir de la question: "La recherche est [...] possible parce que le savoir de la question anticipe celui de la réponse » (Fabre, 2007, p. 71). Lorsque l'enseignante demande à propos du soldat de la Première Guerre mondiale : "Est-ce qu'il disait tout dans sa lettre? », elle formule un angle d'analyse nouveau, auquel les élèves n'avaient pas pensé jusque-là, mais qu'ils comprennent parce qu'il anticipe des réponses ${ }^{5}$ disponibles dans leur connaissance du monde social (de ce qu'est un échange épistolaire et plus généralement de ce qu'est un témoignage ${ }^{6}$ ). Lorsque l'enseignante fait comparer deux scènes du film sur les prémices de la Révolution (l'une en 1788 sans révolte et l'autre un an après avec révolte des paysans), elle vise à poser un problème à une échelle pertinente - celle du moment de décision du groupe - pour explorer les différentes dimensions du film laissées dans l'ombre par la première lecture réaliste des élèves : ils décident la révoltent parce qu'ils ont été informés, parce qu'ils sont nombreux et cela donne du courage, etc. À l'échelle d'une situation humaine, les élèves parviennent à produire des questionnements. Ainsi, lorsque l'enseignante qui fait travailler ses élèves sur la figure 1 permet des échanges qui vont jusqu'à questionner les interprétations proposées par les autres, le document tend à être dégagé de la seule appréhension réaliste parce que plusieurs interprétations apparaissent possibles aux yeux des élèves, comme le manifeste cette intervention d'élèves citée par Y. Le Marec (2010, p. 129) : "Mais un homme s'est rebellé car il n'accepte plus que les riches paient ni impôts ni corvées, mais comment tu peux le savoir Coline ? C'est pas sur l'image ». À nouveau, un détour par l'épistémologie nous fournit des pistes de compréhension des faits didactiques ainsi établis.

En effet, ce qui donne du sens à la recherche documentaire pour les historiens, réside dans la formulation d'un problème préalable et pertinent dans la discipline. Pour eux, 
pas de recherche historique des faits dans la documentation sans les inscrire dans une argumentation (Prost, 2002), et une telle argumentation peut difficilement se tenir à la seule échelle contextuelle: "chaque configuration sociale est le résultat de l'interaction d'innombrables stratégies individuelles: un entrelacement que seule l'observation rapprochée permet de reconstruire " (Ginzburg, 2010 [2006], p. 401). Il nous faut donc reformuler notre problème didactique. Il ne s'agit pas de choisir entre le contexte passé et les actions singulières du document mais de reconstruire la situation dans laquelle ils se croisent $^{7}$. Dans le cadre de la classe, ce type d'activité peut être organisé par le travail didactique du professeur.

\section{Confronter les composantes des documents à un problème de connaissance d'une situation passée}

Un cas exceptionnel peut sans doute mieux faire comprendre les enjeux fondamentaux de cette relation épistémologique entre problème de connaissance du passé et analyse critique des documents comme étude d'une situation. D'un point de vue théorique, on peut résumer l'enjeu par l'idée que la connaissance historique est moins la connaissance du passé que celle des traces, toujours situées du passé. Mais rien ne vaut un exemple pour donner du contenu empirique à cette affirmation théorique, surtout lorsque sont en question des pratiques de lecture.

17 Un groupe d'élèves de $4^{\mathrm{e}}$ travaille sur l'évènement " chute de la monarchie ${ }^{8}$ " avec à sa disposition un chapitre de manuel, et en particulier une double page (figure 3) qu'ils utilisent principalement en lisant la partie gauche qui synthétise le cours des évènements, tout en parcourant parfois les documents lorsqu'un renvoi dans le texte de cours en fait mention (Doussot, 2010).

Figure 3 : la double page de manuel utilisée pour « la chute de la monarchie »

\section{La fin de la monarchie (1791-1792)}
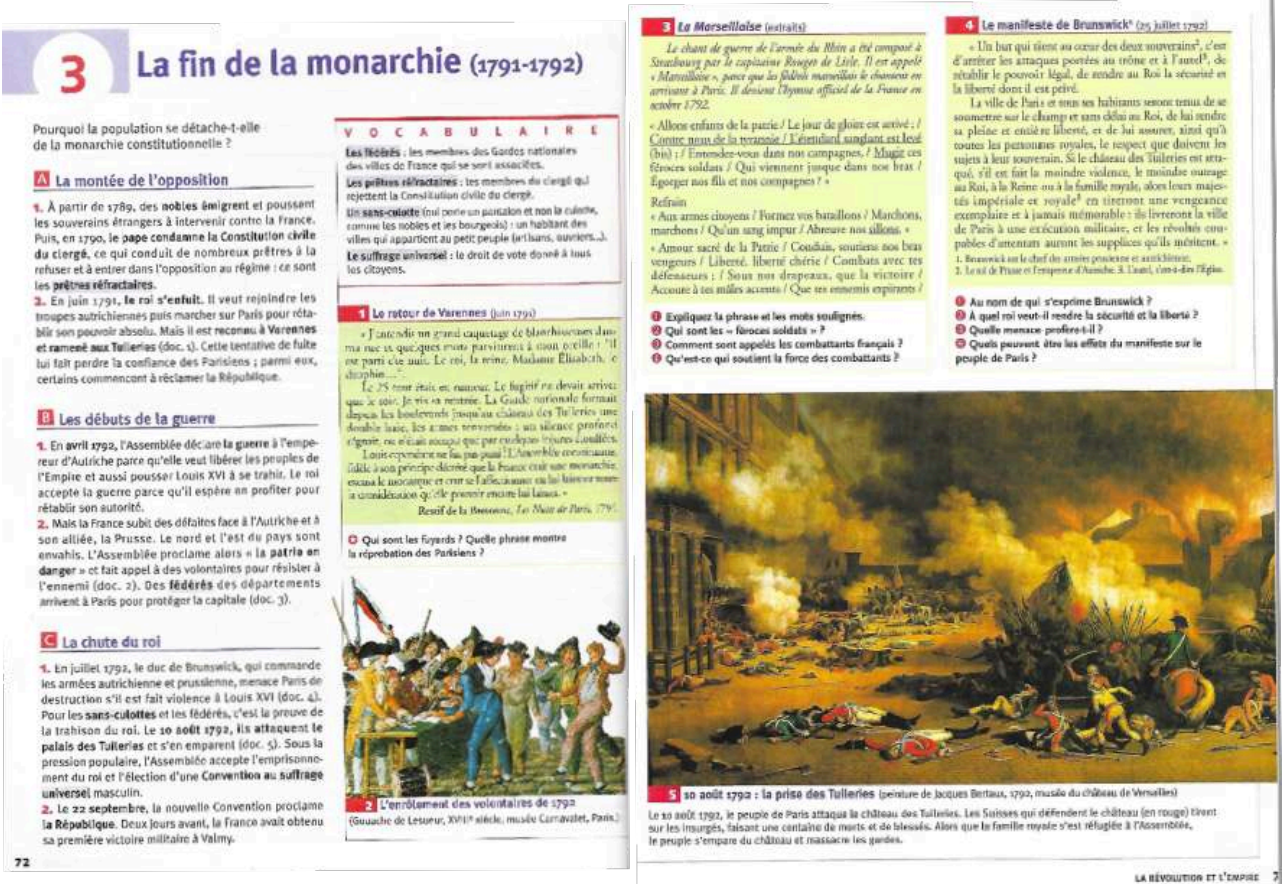
'est que lorsque se pose pour eux un problème collectif à régler pour remplir le tableau-consigne (qui demande de lister les explications possibles à l'arrestation du roi - colonne de gauche - et de justifier pourquoi ces explications répondent à la question - colonne de droite) que les élèves sortent de leur lecture réaliste du cours du manuel et qu'ils entrent dans la lecture d'un des documents de la page de droite de ce même manuel. C'est un problème de chronologie qu'ils élaborent presque à leur insu et qui est à la fois pertinent historiquement pour penser les causalités, et pertinent pour eux parce que la chronologie constitue un critère de réussite en classe d'histoire : pourquoi les révolutionnaires n'ont-ils pas arrêté le roi dès la première cause de mécontentement envers celui-ci (la fuite et la déclaration de guerre de Louis XVI décrites comme trahison)? Dans ce contexte problématique le document «Manifeste de Brunswick » (sur la figure 3) prend sens pour les élèves par rapport au texte de cours du manuel parce qu'il manifeste, littéralement, pour les révolutionnaires la trahison même qu'a constitué la fuite ou la déclaration de guerre qui n'était pas initialement pensée comme telle ${ }^{9}$. Mais ce rapport entre deux temporalités - celle du roi qui trahit et celle des révolutionnaires qui ne savent pas immédiatement établir que le fuite est une trahison - n'est pas explicitée par le cours du manuel, alors que seule cette différenciation des temporalités peut donner sens au problème chronologique des élèves. La lecture de la déclaration de Brunswick, chef des armées ennemies, est alors lue dans le document de la page de droite du manuel pour ce qu'elle est: une menace envers les révolutionnaires, car sa nature de menace constitue un indice de l'hypothèse $\mathrm{du}$ complot et non plus seulement une information parmi d'autres que fournit l'appréhension "externe" de ce document. Le style direct qui se mêle au style explicatif des élèves (voir note 7) dans cette phase montre comment la lecture du manifeste est «jouée» pour rendre compte de son contenu et de son intention simultanément. Les élèves se représentent les enjeux de la situation - comme une scène - qui prend sens avec ce document en tant qu'il en constitue une action centrale. Mais cela n'est possible que parce que les élèves ont à résoudre un problème de chronologie et de causalité qui institue l'information sur la nature du document en indice.

Cet exemple souligne la double dimension des connaissances des élèves nécessaires pour entrer dans une telle lecture des documents (ou ensemble de documents) composites : connaissance sur l'évènement mais aussi connaissance sur les critères de validité des discours à tenir dans la discipline (les types de raisonnement, notamment chrono-causal). Ce sont ces connaissances qui fournissent la matière première indispensable à la construction d'un problème susceptible de conduire à une enquête documentaire valable dans la discipline. La spécificité de ce cas, exceptionnel du fait de la construction autonome par les élèves d'un problème historique et à leur portée, nous ouvre à la compréhension des conditions de construction de situations didactiques favorisant cette problématisation, conditions susceptibles de guider les choix des enseignants. Les études évoquées précédemment fournissent des pistes en ce sens qu'elles permettent de poursuivre l'exploration des spécificités disciplinaires de lecture de documents composites en histoire.

20 Le cas du soldat de la Première Guerre mondiale est particulièrement heuristique de ce point de vue. La potentialité révélée par la première version de la séquence, évoquée précédemment, a ensuite été mise à profit pour organiser de nouvelles versions régulées par le jeu explicite entre composantes du dossier documentaire ${ }^{10}$. Une 
deuxième version oppose, dans le dispositif de questionnement des élèves, la lettre et le journal de l'unité qui tous deux donnent à voir des informations différenciées sur la réalité des combats, en particulier le fait de donner la mort n'est pas évoqué dans la lettre tandis qu'il l'est dans le journal. L'enjeu de lecture est clair : les deux textes se ressemblent dans leur contenu, en termes d'informations données sur l'expérience du front, mais seule l'appréhension de leur composante factuelle (nature, auteur, destinataire, etc.) permet de donner un sens aux écarts entre leurs contenus. En demandant aux élèves de rechercher cet écart entre les informations données par l'un et l'autre de ces documents, on focalise leur attention sur un problème historique en ce qu'il porte à la fois sur la connaissance de ces traces du passé (ce qu'elles disent et ne disent pas, en fonction de leur situation énonciative respective) et sur le phénomène lui-même (l'expérience des tranchées). Autrement dit, c'est la mise en tension des composantes différenciées - du point de vue énonciatif (sous-officier pour sa hiérarchie vs mari pour sa femme), et sémiotique (lettre vs journal organisé en tableau et listes) $\mathrm{du}$ dossier documentaire et de chacun des documents qui devient un levier pour construire le savoir nouveau sur le thème (l'expérience du front) et sur les documents eux-mêmes. En histoire, la dimension composite des documents est donc potentiellement une source de compréhension du passé et des traces qui subsistent.

Une troisième version de la séquence sur ce soldat s'appuie quant à elle sur l'identification contingente d'une difficulté de lecture de la lettre du soldat: dans chacune des classes expérimentées aucun des élèves n'a évoqué le dernier paragraphe de sa lettre à sa famille. On peut expliquer cette absence par la rupture que le paragraphe opère avec le reste du texte et avec les explications mobilisées par les élèves (autour de la souffrance et du statut de victime du soldat), puisque le soldat y explique en substance qu'il oublie les carnages mais ne pourra jamais oublier les destructions matérielles («des chaumières» notamment). Nous choisissons de confronter explicitement les élèves à ce constat d'une lecture systématiquement partielle : que veut dire le soldat dans ce paragraphe laissé de côté par la première lecture ? Dès lors se pose un problème historique : de modalité de lecture du document (pourquoi a-t-on "oublié» le paragraphe en question?) et d'interprétation de l'expérience de guerre (comment est-il possible que le soldat dise cela ?). Là encore, le dispositif didactique conduit à une lecture renouvelée du document par mise en tension de ses composantes variées sur le plan énonciatif et sémiotique. Mais plus encore, il conduit à identifier un processus de décomposition/recomposition du document. Le travail des documents dans le cadre d'une analyse réaliste des élèves les conduit, nous l'avons signalé au début, à prélever des informations sans souci des positionnements énonciatifs ni des systèmes sémiotiques dans lesquels ils s'inscrivent. Par ce travail de décomposition, les élèves peuvent isoler des éléments qui ne font pas sens pour eux avec les connaissances qui sont à leur disposition, comme dans le cas de ce paragraphe de la lettre. Autrement dit, pour les élèves, les documents sont composites sous des formes qui ne correspondent pas directement aux découpages didactiques que nous avons utilisés (voir section 1 du présent article). Ici, c'est le sens de ce qu'écrit ce soldat qui définit un découpage qui pose problème aux élèves, à tel point qu'ils ignorent tous l'un des paragraphes, sans que celui-ci ne se distingue d'une autre manière. La mise au travail de ce découpage interne au texte conduit certains élèves à faire des hypothèses explicatives (le soldat est "matérialiste», ou bien il vise ainsi à "atténuer les horreurs " qu'il a décrites dans le début de sa lettre), dont certaines poussent à reconsidérer la composante factuelle de la lettre, c'est-à-dire la situation singulière de 
ce soldat écrivant : la légende dit qu'il est boulanger, qu'il habite à la campagne, peutêtre peut-on en inférer qu'il accorde davantage de valeur à ce qu'est une maison qu'on peut le faire aujourd'hui parce qu'il a peut-être lui-même construit sa maison ? À nouveau c'est l'écart qualitatif entre les composantes du document qui permet de poursuivre la recherche et, simultanément, d'élucider ce caractère composite.

Ces perspectives didactiques mettent au jour la circularité de ces deux processus. L'élucidation du caractère composite des documents se fait au cours de l'enquête menée sur les traces du passé, qui est elle-même soumise à des variations liées à ce caractère composite. Cela correspond à un double processus qui n'est pas propre à la discipline historique. Comme le soulignent A. Tricot, G. Sahut \& J. Lemarié, 2016 (2016, p. 62), « le besoin étant situé, la pertinence l'est aussi. Comme le besoin d'information évolue au cours de la recherche (Rouet et Tricot, 1995), les jugements de pertinence évoluent eux aussi durant celle-ci ». Dans le cas de l'histoire, cela conduit à une reconstruction du questionnement initial en problème historique nouveau. Pour les élèves, la question initiale qui portait sur l'expérience des tranchées (contexte a priori) est peu à peu remplacée par la compréhension des relations entre le front et l'arrière ${ }^{11}$ (contexte reconstruit, adapté aux contraintes qu'impose la documentation étudiée). Ce processus se produit par la focalisation sur la situation d'écriture de ce soldat (à quoi pense-t-il en écrivant à sa famille ?), et non plus sur une image vague de la tranchée. Cette problématisation progressive repose sur une exploration elle-même nouvelle des documents et de leurs multiples composantes qui tendent à entrer en tension au fil des explorations. Cependant, pour que les élèves accèdent à des savoirs de nature proprement historique dans ce processus essentiel (parce que fondés sur l'exploration des explications possibles portées par les sources), le jugement produit sur les documents et le passé en question n'est pas «parfaitement subjectif » (Tricot, Sahut \& Lemarié, 2016., 2016, p. 63). Du point de vue disciplinaire, il est au contraire le résultat d'activités d'enquête contrôlées collectivement parce que contrôlables: par la focalisation sur une situation qui rend accessible une appréhension critique des comportements humains entre contraintes et incertitudes. En cela, la recherche documentaire, lorsqu'elle s'inscrit dans une discipline à visée scientifique, ne peut être réduite à une compétence générale, c'est-à-dire imprécise, de recherche documentaire.

\section{Conclusion}

23 Les difficultés de lecture des documents composites que l'on peut observer en classe pourraient conduire à une élaboration trop rapide de solutions d'enseignement. Le détour par la recherche didactique sert justement à suspendre la production de solutions en passant d'un objet du monde scolaire - les différents types de documents possibles et leurs différentes composantes - à un objet de recherche construit sur la base, non seulement des documents, mais aussi des usages effectifs dans différentes situations. C'est ce que le retour sur certaines études didactiques produites dans le cadre d'autres objectifs a permis au cours de cette étude. Elle met en lumière, par le biais d'un regard épistémologique, que la distinction entre information et indice est symbolique de l'importance peu visible du rôle du questionnement dans les processus de lecture de documents (et de dossiers documentaires) composites en classe d'histoire. Ainsi saisi au sein d'un processus de problématisation historique, la lecture des documents composites révèle-t-elle à la fois des découpages initialement invisibles 
dans les catégories ordinaires de l'analyse des documents, et le rôle moteur que ces différences de nature entre composantes peuvent jouer dans l'apprentissage de l'histoire et le processus même de lecture.

Sur cette base, et en référence aux travaux en information-communication sur les processus de recherche documentaire, il est même possible de pousser plus loin encore l'approche disciplinaire de la compétence correspondante. Si elle est incontestablement liée à la connaissance des contenus de savoir en jeu, et donc loin d'être transversale, c'est-à-dire potentiellement acquise une fois pour toute, elle renvoie toujours simultanément à la connaissance d'un fonctionnement et d'un contenu disciplinaires. Cette connaissance peut être celle de l'histoire ou de la documentation médiatique. C'est en tout cas ce que laisse entrevoir une récente étude comparative menée par deux didacticiens de l'histoire américain (Wineburg \& McGrew, 2017), qui confronte des étudiants d'histoire de premier cycle, des historiens chevronnés et reconnus, et des spécialistes du fact-checking sur internet à propos de l'actualité, à des pages internet d'information composites. Les spécialistes du fact-checking se détachent largement des deux autres catégories, et les historiens ne sont pas vraiment plus performants que les étudiants. La capacité à vérifier de l'information dépend bien à la fois des connaissances du domaine (médiatique dans cette expérimentation) et des types de problèmes posés. Ceux des historiens ne sont pas ceux des lecteurs de sites internet.

\section{BIBLIOGRAPHIE}

BAZIN, J. (2008). Des clous dans la Joconde. L'anthropologie autrement. Toulouse : Éd. Anacharsis.

BLOCH, M. (1997) [1949]. Apologie pour l'histoire ou Métier d'historien. Paris : A. Colin.

BOUBÉE, N. \& TRICOT, A. (2011). Activité informationnelle juvénile. Paris : Lavoisier.

CARIOU, D. (2016). « Information ou indice? Deux lectures d'une image en classe d'histoire ». Revue française de pédagogie 197, p. 63-78. En ligne : https://journals.openedition.org/rfp/5160.

Doussot, S. (2010). « Pratiques de savoir en classe et chez les historiens : une étude de cas au collège ». Revue française de pédagogie 173, p. 85-104. En ligne : https://journals.openedition.org/ $\mathrm{rfp} / 2586$.

Doussot, S. (2017). « Modélisation des problématisations historiques en classe et chez les historiens ». Recherches en éducation 29. En ligne : http://www.recherches-en-education.net/IMG/ pdf/REE-no29.pdf.

DOUSSOT, S. \& VEZIER, A. (2014). « Des savoirs comme pratiques de problématisation : une approche socio-cognitive en didactique de l'histoire ». Éducation et didactique 8 (3), p. 111-139. En ligne : https://journals.openedition.org/educationdidactique/2097.

DOUSSOT, S. \& VEZIER, A. (2015). « Débat historiographique sur le Grande Guerre et étude de cas en histoire : des références pour la classe ? ». Didactica Historica 1, p. 77-82. 
FABRE, M. (2007). « Des savoirs scolaires sans problèmes et sans enjeux. La faute à qui ? ». Revue française de pédagogie 161, p. 69-78. En ligne : https://journals.openedition.org/rfp/823.

FINK, N. (2014). Paroles de témoins, paroles d'élèves. La mémoire et l'histoire de la Seconde Guerre mondiale, de l'espace public au monde scolaire. Berne/Berlin/Bruxelles : P. Lang.

GINZBURG, C. (2010) [2006]. Le fil et les traces : vrai faux fictif. Trad. de l'italien par M. Rueff. Lagrasse : Verdier.

LE MAREC, Y. (2010). « Construction de problèmes en histoire et régulation de l'activité de l'enseignant : une étude de cas à l'école primaire ». Le cartable de Clio 10, p. 121-135.

MEKNASSI (el), É. (2014). Didactique de l'étude de cas en histoire. Mémoire de master en métiers de l'éducation et de la formation : Inspé Académie de Nantes. En ligne : http://dumas.ccsd.cnrs.fr/ dumas-01222541/document.

PROST, A. (1996). Douze Leçons sur l'histoire. Paris : Édition du Seuil.

PROST, A. (2002). « Argumentation historique et argumentation judiciaire ». In : Fornel, M. \& Passeron J.-C. (éd.), L'argumentation, preuve et persuasion. Paris : Éditions de l'EHESS, p. 29-47. RIC@UR, P. (2000). La mémoire, l'histoire, l'oubli. Paris : Éditions du Seuil.

TRICOT, A., SAHUT, G. \& LEMARIÉ, J., (2016). Le document : communication et mémoire. Bruxelles : De Boeck.

TUTIAUX-GUILlon, N. (2008). «Interpréter la stabilité d'une discipline scolaire : l'histoiregéographie dans le secondaire français ». In : Audigier F. \& Tutiaux-Guillon N. (dir.), Compétences et contenus. Louvain-la-Neuve : De Boeck, p. 117-146.

WINEBURG, S. \& MCGREW, S. (2017). Lateral Reading: Reading Less and Learning More When evaluating Digital Information. Stanford History Education Group Working Paper $\mathrm{n}^{\circ}$ 2017.A1 : Stanford : Stanford University Graduate School of Education.

\section{ANNEXES}

\section{Références iconographiques}

Fig. 1. (1789) Le Temps passé les plus utiles etaient foulés aux pieds : taille, impôts et corvées. Estampe. Paris. Appartient à : Recueil. Collection Michel Hennin. Estampes relatives à l'Histoire de France. Tome 120, Pièces 10490-10613. Domaine Publique. Source : Bibliothèque nationale de France/Gallica. En ligne : https://gallica.bnf.fr/ark:/12148/ btv1b84110012.item\#.

Fig. 2. Ministère des Armées. Service historique de la Défense (SHD). $346^{\mathrm{e}}$ régiment d'infanterie J.M.O. 26 N 757/2. 1er septembre 1915-1931-décembre 1916, p. 38. Référence : SDH - Vincennes, GR 26 N 7570020038 En ligne : https:// www.memoiredeshommes.sga.defense.gouv.fr/fr/arkotheque/inventaires/ ead_ir_consult.php?fam=3\&ref=6\&le_id=2346.

Fig. 3. Manuel d'Histoire Géographie 4e (2002). Paris : Éditions Hatier, p. 72-73.

- Doc. 1. RÉTIF DE LA BRETONNE, N.-E. (1788). La Nuit de Paris. Londres. En ligne : https:// gallica.bnf.fr/ark:/12148/bpt6k6423512k. 
- Doc. 2. LESUEUR, J.-B. (ca. 1792-1793). Après la trahison de Dunouriez qui a voit livré aux Ennemis une partie de l'armée [...]. Gouache. Paris : Musée Carnavalet. Domaine public. En ligne : https://commons.wikimedia.org/wiki/File:Levee_en_masse.jpg. - Doc. 3. Rouget de Lisle (1792). La Marseillaise. Paroles. En ligne : https:// www.elysee.fr/la-presidence/la-marseillaise-de-rouget-de-lisle.

- Doc. 4. Charles-Guillaume-Ferdinand, duc de Brunswick-Lunebourg (1792). Le Manifeste de Brunswick. Domaine public. En ligne : https://fr.wikisource.org/wiki/ Manifeste_de_Brunswick.

- Doc. 5. DUPLESSIS-BERTAUX, J. (1993). La prise des Tuileries le 10 août 1792. Tableau. Paris : Musée du château de Versailles. Domaine public. En ligne : https:// commons.wikimedia.org/wiki/File:Tuileriensturm.jpg?uselang=fr.

\section{NOTES}

1. Document comme fac-similé de la trace notamment.

2. Comme le formule J. Bazin (2008, p. 356), à propos du travail de l'ethnographe ou de l'historien, «je risque de fort mal raconter la bataille d'Austerlitz si je ne sais pas comment on fait la guerre à cette époque ; mais pour apprendre comment on fait la guerre à cette époque la bataille d'Austerlitz peut me servir de leçon, pour autant que j'en ai un récit exact ».

3. Pour compléter cet exemple on peut également se reporter à l'étude de Meknassi (2014) à propos de l'enseignement de la guerre d'Algérie en classe de $3^{\mathrm{e}}$.

4. Intitulé 1788 , il retrace la vie d'un village de Touraine entre l'été 1788 et l'été 1789.

5. «Toute problématisation exige donc un savoir questionner, un savoir des questions, complètement distinct du savoir des réponses » (Fabre, 2007, p. 71).

6. Voir sur ce point précisément la potentialité didactique de l'étude des témoignages de Fink (2014) fondée sur l'expérience universelle de la relation testimoniale que Ricœur (2000, p. 26) pointe comme «structure fondamentale de transition entre la mémoire et l'histoire ».

7. «Il est remarquable que le rapport entre la dimension microscopique et la dimension contextuelle soit devenu [...] le principe organisateur de la narration [en histoire]» (Ginzburg, 2010 [2006], p. 401-402).

8. Entre la tentative de fuite de Louis XVI en juin 1791 et son arrestation le 10 aout 1792.

9. Voici comment cela se manifeste dans leurs échanges retranscrits, jusqu'à l'identification du complot qui manifeste l'écart temporel entre ceux qui savent et ceux qui ignorent :

$121 \mathrm{~A}$ : ouais mais au début le peuple il le sait pas, au début le peuple y croit que c'est une guerre qu'est déclarée à la France et après...

122 Jo : et en fait y...

123 A : ... on s'aperçoit, parce que le... le chef des autrichiens y dit, euh, si vous laissez pas les pouvoirs à Louis XVI, euh, j'envahis la capitale.

$124 \mathrm{Jo}$ : OK, ouais. En fait y se rend compte que c'est une magouille, quoi.

125 A : Ouais. Comment ça se dit une magouille sans être familier.

$126 \mathrm{~J}$ : Un complot. 
10. Il est composé de la lettre à sa famille, d'un extrait du journal de son unité dans les jours qui précède sa mort à Verdun, d'un extrait de son livret militaire qui indique des éléments de son identité une photo, et d'un plan de la situation du champ de bataille à Verdun dans lequel il trouve la mort. Pour plus de détails voir (Doussot \& Vézier, 2015). 11. Notamment la part indicible de l'expérience des combats.

\section{RÉSUMÉS}

Le travail documentaire fait partie de l'essence même de l'approche historique, les traces du passé étant par nature composites. Transposé en histoire scolaire à travers «l'étude de documents ", ce travail se focalise généralement sur les différentes composantes des documents en vue de confronter ce qu'ils disent ce qu'ils sont. Mais cette transposition modifie radicalement la nature épistémologique du travail de lecture des documents composites par les élèves vis-à-vis à la visée scientifique. L'article prend appui sur l'historiographie et l'épistémologie de l'histoire, sur la base de quelques cas, pour mettre au jour des conditions disciplinaires de gestion de l'hétérogénéité des registres énonciatifs et des systèmes sémiotiques lors de l'acte de lecture des documents. Il apparait qu'il serait plus aisé pour les élèves de lire des documents composites lorsque ces derniers constituent des traces d'une même situation socio-historique.

Documentary work is part of the very essence of the historical approach, traces of the past being by nature composite. Transposed into school history through the "study of documents", this work generally focuses on the different components of documents in order to compare what they say to what they are. But this transposition radically modifies the epistemological nature of the students' work of reading composite documents in relation to the scientific aim. On the basis of a few cases, the article draws on the historiography and epistemology of history to reveal, disciplinary conditions for managing the heterogeneity of enunciative registers and semiotic systems during the act of reading documents. It appeared that it would be easier for students to read composite documents when they constitute traces of the same socio-historical situation.

\section{INDEX}

Keywords : history, didactics, problem-based learning, situation, clue

Mots-clés : histoire, didactique, problématisation, situation, indice

\section{AUTEUR}

\section{SYLVAIN DOUSSOT}

Inspé de l'académie de Nantes, Cren, F-44300 Nantes, France. 